Eronildo Felisberto 1

Eduardo Freese de Carvalho 2

Ruben Schindler Maggi 1

Isabella Samico 1

\section{Avaliação do processo de implantação da estratégia da Atenção Integrada às Doenças Prevalentes da Infância no Programa Saúde da Família, no Estado de Pernambuco, Brasil}

\author{
Implementation process evaluation \\ of the Integrated Management Childhood IIIness \\ strategy in the Family Health Program, \\ Pernambuco State, Brazil
}

1 Instituto Materno Infantil de Pernambuco.

Rua dos Coelhos 300 ,

Recife, PE 50070-550, Brasil.

eronildo@imip.org.br

2 Núcleo de Estudos

em Saúde Coletiva,

Centro de Pesquisas

Aggeu Magalhães,

Fundação Oswaldo Cruz.

Rua dos Coelhos 450 ,

Recife, PE 50070-550, Brasil.

\begin{abstract}
This evaluative study attempts to define the contextual determinants of the degree of implementation in the Integrated Management of Childhood Illnesses strategy in Pernambuco State, Brazil. A total of 33 Family Health Program teams were selected from 10 municipalities. In order to define the degree of implementation, the study used a specific score system and the process indicators provided by the strategy. Municipalities were classified as critical, unsatisfactory, and acceptable. All municipalities except for one presented an acceptable degree of implementation in relation to the structure. In relation to process evaluation, two municipalities received one follow-up visit and their scores were unsatisfactory. Four municipalities received two visits, and one was classified as unsatisfactory on the second follow-up visit, while another presented a critical score on the first follow-up visit. Among four municipalities that received three follow-up visits, one was classified as unsatisfactory in the three evaluations. Four municipalities achieved mean unsatisfactory scores, when considering the mean scores acquired in the three follow-up visits. There was no association between the organizational context and degrees of implementation.
\end{abstract}

Key words Child Welfare; Family Health Program; Evaluation

Resumo Estudo avaliativo que procura definir os determinantes contextuais do grau de implantação da estratégia Atenção Integrada às Doenças Prevalentes da Infância, no estado de Pernambuco. Selecionou-se 33 unidades do Programa Saúde da Família, de dez municípios. Para definir o grau de implantação, utilizou-se um sistema de escores específico e os indicadores de processo normatizados pela estratégia, classificando os municípios nos níveis Crítico, Insatisfatório e Aceitável. Os municípios, com uma exceção, apresentaram nível aceitável em relação à estrutura. Quanto à avaliação do processo, dois receberam uma visita de seguimento e os escores encontraram-se no nível insatisfatório. Quatro receberam duas visitas, com um deles classificado no nível insatisfatório no 2 o seguimento e outro apresentando nível crítico no lo seguimento. Em quatro municípios que receberam três visitas, um obteve escores no nível insatisfatório nas três avaliações. Observa-se que quatro municípios atingiram escores médios, que os classificam no nível insatisfatório quando se consideram as médias dos escores obtidos nas 3 visitas de seguimento. Não foi encontrada associação entre contexto organizacional e os graus de implantação. Palavras-chave Bem-Estar da Criança; Programa Saúde da Família; Avaliação 


\section{Introdução}

A estratégia da Atenção Integrada às Doenças Prevalentes da Infância (AIDPI), apresenta um novo enfoque de abordagem à saúde da criança no primeiro nível de assistência. Avalia de maneira sistemática os principais fatores que afetam a saúde das crianças, integrando ações curativas com medidas de prevenção e promoção. Introduz o conceito de integralidade, surgindo como alternativa aos programas específicos já existentes (Benguigui et al., 1997).

Inicialmente introduzida em países africanos e asiáticos, a estratégia, denominada Integrated Management of Childhood Illness (IMCI), foi submetida a um pré-teste na Etiópia em 1994, e até 1996 alguns países daqueles continentes haviam iniciado o processo de implantação da proposta (WHO, 1997).

Visando a incorporação da estratégia como política de saúde fundamental dos países em desenvolvimento, para o alcance das metas da Cúpula Mundial em Favor da Infância, a Organização Pan-Americana da Saúde (OPAS) desencadeia na América Latina um processo, em 1996, objetivando promover a participação de organismos internacionais, que contribuem para acelerar a efetiva implantação da AIDPI no continente (OPS, 1998).

O Ministério da Saúde do Brasil (MS) adota a AIDPI a partir de 1995, e em 1996 promove a adaptação do material instrucional às normas nacionais. O Estado de Pernambuco, junto com os estados do Pará, Ceará e Sergipe, inicia, nesse ano, o processo de implantação da estratégia em municípios com taxas de mortalidade infantil acima de 40/1.000 nascidos vivos (Felisberto et al., 2000).

A universalização do acesso, a integralidade da assistência e a regionalização, hierarquização e descentralização dos serviços de saúde, princípios do Sistema Único de Saúde (SUS), prevêem a busca de metodologias de investigação que permitam a transformação da realidade dos serviços e sua adaptação às melhorias necessárias.

Para Contandriopoulos et al. (1994), uma intervenção, qualquer que seja, pode sofrer dois tipos de avaliação: (a) pode-se estudar cada componente da intervenção em relação a normas e critérios (avaliação normativa) e (b) pode-se examinar as relações que existem entre os diferentes componentes da intervenção (pesquisa avaliativa).

Segundo Denis \& Champagne (1997), a análise de implantação visa definir a influência dos fatores contextuais nos efeitos e no grau de implantação da intervenção. Esses autores apresentam proposta de um quadro conceitual para atingir o objetivo acima exposto. De acordo com essa proposição, que chamam de modelo político e contingente, “... os diferentes atores organizacionais podem apoiar a implantação de uma intervenção se virem nela um meio de atualização de suas estratégias fundamentais" (Denis \& Champagne, 1997:67).

"Segundo este modelo, o processo de implantação de uma intervenção deve, antes de tudo, ser abordado segundo uma perspectiva política. Ele sofre, todavia, as pressões de caráter estrutural, isto é, as características estruturais de uma organização que funcionam em sinergia ou em antagonismo na atualização das estratégias dos atores" (Denis \& Champagne, 1997:67).

É baseado nessa proposição que se procurou, também, situar o método utilizado neste estudo. A investigação proposta tenta, assim, se fundamentar no princípio da garantia da qualidade, cujos métodos, sugeridos inicialmente por Donabedian (1990), podem ser divididos em três tipos de abordagem: estrutural, de processo e de resultado (Vuori, 1988).

Este estudo propõe-se, também, a abordar um dos múltiplos enfoques que podem direcionar o processo de avaliação de serviços de saúde: a análise dos determinantes contextuais do grau de implantação de programas, um dos três componentes que, segundo Denis \& Champagne (1997:55), "apóia a análise de implantação de programas". Os outros dois são: a análise das variações da implantação na sua eficácia, e a análise da interação entre o contexto da implantação e a intervenção nos efeitos observados.

Dessa forma, tomando como referencial teórico as abordagens conceituais trabalhadas por Hartz (1997), procura-se responder as duas principais questões levantadas neste estudo:

Qual o grau de implantação alcançado pela estratégia AIDPI no Estado de Pernambuco, considerando-se os resultados da avaliação de estrutura e processo de suas atividades?

Como o grau de implantação da estratégia AIDPI no Estado de Pernambuco é condicionado pelo contexto organizacional no nível municipal?

\section{Material e método}

O modelo utilizado para o estudo é do tipo avaliativo, que incorpora a avaliação normativa em seus componentes de estrutura e de processo, e promove uma pesquisa avaliativa para definir os determinantes contextuais do grau de implantação da intervenção.

Selecionou-se 33 unidades do Programa Saúde da Família (PSF), cujos profissionais foram 
treinados na estratégia AIDPI de dez municípios do Estado de Pernambuco, nos quais a Secretaria Estadual de Saúde (SES-PE) realiza o monitoramento desde agosto de 1998, e que até dezembro de 1999 deveriam ter recebido três visitas de seguimento.

Para descrever a implantação, utilizaram-se os resultados das visitas de seguimento, componente da estratégia, cujos questionários analisados em Epi-Info (Dean et al., 1994), foram consolidados previamente em relatórios oficiais.

Para a avaliação da estrutura, utilizou-se o mesmo banco de dados e as variáveis selecionadas foram: medicamentos, vacinas, equipamentos/insumos e recursos humanos.

Para definir o grau de implantação e permitir a avaliação do processo de implantação, utilizaram-se dois métodos distintos. O primeiro, denominado de sistema de escores, desenvolvido pelo autor, estabelece um mecanismo que permite atribuir aos municípios uma pontuação que os classifica em graus e, ainda, permite conhecê-la por variável estudada. O segundo, utiliza os indicadores de processo normatizados pelo MS e classifica os municípios por meio da média aritmética dos porcentuais obtidos para cada indicador (MS, 2000). As variáveis escolhidas são porcentuais de: profissionais de nível superior capacitados na AIDPI, casos classificados corretamente, casos orientados corretamente sobre alimentação, casos referenciados com urgência adequadamente, unidades de saúde com medicamentos padronizados para a AIDPI e profissionais que receberam no mínimo uma visita de seguimento semestral após a capacitação na estratégia.

Para criar o sistema de escores, utilizaramse as seguintes variáveis e os respectivos pesos que foram sugeridos pelo autor de acordo com a relação que cada uma guarda com o estado de saúde da criança: classificação correta (2), tratamento correto (2), recomendou vacinas (1), recomendou alimentação $(0,5)$, recomendou retorno $(0,5)$, verificou preenchimento do cartão $(0,5)$, seguiu a sistematização do atendimento $(0,5)$, compreensão satisfatória do problema (1), compreensão satisfatória sobre o tratamento (1), compreensão satisfatória sobre outras orientações $(0,5)$ e compreensão satisfatória sobre a necessidade de retorno $(0,5)$. A ponderação toma ainda como base, os instrumentos: Condições de Eficiência dos Serviços de Atenção Materno-Infantil e Roteiro de Avaliação dos Programas: Programa de Assistência Integral à Saúde da Mulher (PAISM), Programa de Assistência Integral à Saúde da Criança (PAISC) e Programa Saúde do Adolescente (PROSAD) (MS, 1994; OPAS/OMS, 1987). A pontuação máxima obtida pelo conjunto dessas variáveis é 10, sendo conseguida a partir dos percentuais de positividade alcançados por cada variável.

A pontuação obtida por cada um dos municípios nesta etapa denominou-se de nota final do caso e, a ela, somou-se a pontuação que foi denominada nota final dos insumos, resultado dos escores individuais das variáveis de estrutura citadas a seguir com seus respectivos pesos individuais: medicamentos (1), vacinas (1) e equipamentos (1), cuja pontuação máxima definida é 10.

Para se obter a pontuação final com vistas a definir o grau de implantação, obteve-se a média ponderada dessas últimas duas variáveis compostas, atribuindo-se a elas os pesos 2 para a nota final do caso e 1 para a nota final dos insumos.

O grau de implantação foi definido como: crítico (C) para os valores de 0 a 4,9; insatisfatório (I) para os de 5,0 a 7,9 e aceitável (A) para 8,0 e mais. Esta foi utilizada tanto para classificação com o sistema de escores como para a classificação final, após a aplicação dos indicadores de processo (Cosendey, 2000; Hartz, 1997; Ojeda, 1992; SES-PE, 1996).

Para a análise de implantação, relacionando o grau de implantação e o contexto organizacional, utilizou-se a abordagem qualitativa com o recurso da entrevista com atores chaves das gestões municipais e das unidades, e a análise de documentos oficiais. Realizaram-se 10 entrevistas com os coordenadores do PSF e utilizaram-se os resultados de 146 entrevistas com profissionais das unidades, constantes do banco de dados das visitas de seguimento, realizadas em momentos distintos, ou seja, 66 na $1 \underline{a}$, 52 na $2 \underline{a}$ e 28 na $3 \underline{a}$ visita.

As variáveis contextuais estudadas são: tipo de gestão municipal, qualificação dos gestores, registro em atas do Conselho Municipal de Saúde, plano de trabalho referente à estratégia, recursos financeiros empregados, relatórios sistemáticos de supervisão das unidades, simultaneidade das coordenações do PSF e da AIDPI e mudanças dos atores locais.

\section{Resultados}

Na Tabela 1 observa-se que os municípios apresentam um nível aceitável em relação à estrutura, com exceção do município de Passira, onde o nível é insatisfatório $(6,5)$. Já os municípios de Água Preta, Bezerros e Cabo de Santo Agostinho, embora apresentem escores que os classifiquem no nível insatisfatório, estes escores se aproximam do nível aceitável (7,5, 7,9 e 7,8). 
Tabela 1

Grau de implantação dos municípios avaliados segundo visitas de seguimento.

\begin{tabular}{|c|c|c|c|}
\hline & \multicolumn{3}{|c|}{ Visita de seguimento } \\
\hline & $\begin{array}{l}\text { Escore de caso } \\
\text { do manejo }\end{array}$ & $\begin{array}{l}\text { Escore final } \\
\text { dos insumos }\end{array}$ & Grau de implantação \\
\hline \multicolumn{4}{|c|}{ Agrestina } \\
\hline $1 \underline{a}$ & 6,81 & 8,20 & 7,2 \\
\hline \multicolumn{4}{|c|}{ Passira } \\
\hline 1a & 6,87 & 6,50 & 6,8 \\
\hline \multicolumn{4}{|c|}{ Água Preta } \\
\hline 1aa & 8,93 & 7,50 & 8,4 \\
\hline $2 \underline{a}$ & 9,05 & 9,80 & 9,3 \\
\hline \multicolumn{4}{|c|}{ Bezerros } \\
\hline 1ạ & 8,40 & 9,20 & 8,7 \\
\hline $2 \underline{a}$ & 8,01 & 7,90 & 7,9 \\
\hline \multicolumn{4}{|c|}{ Garanhuns } \\
\hline 1a & 8,96 & 8,90 & 9,0 \\
\hline $2 \underline{a}$ & 9,57 & 8,60 & 9,3 \\
\hline \multicolumn{4}{|c|}{ Saloá } \\
\hline 1a & 0,00 & 10,0 & 3,3 \\
\hline $2 \underline{a}$ & 7,36 & 10,0 & 8,2 \\
\hline \multicolumn{4}{|c|}{ Brejão } \\
\hline $1 \underline{a}$ & 9,27 & 10,0 & 9,5 \\
\hline 2a & 8,60 & 10,0 & 9,0 \\
\hline $3 a$ & 9,83 & 10,0 & 9,8 \\
\hline \multicolumn{4}{|c|}{ Cabo } \\
\hline $1 \underline{a}$ & 7,93 & 8,50 & 8,1 \\
\hline 2a & 7,18 & 8,70 & 7,7 \\
\hline $3 \underline{a}$ & 6,76 & 7,80 & 7,1 \\
\hline \multicolumn{4}{|c|}{ Caetés } \\
\hline 1aa & 9,61 & 9,80 & 9,7 \\
\hline 2a & 9,05 & 8,80 & 8,9 \\
\hline $3 \underline{a}$ & 9,33 & 9,30 & 9,3 \\
\hline \multicolumn{4}{|c|}{ Caruaru } \\
\hline 1a & 8,94 & 9,00 & 8,9 \\
\hline 2 a & 9,24 & 9,90 & 9,4 \\
\hline 3ạ & 9,36 & 9,80 & 9,6 \\
\hline
\end{tabular}

Ressalte-se que isso ocorre quando considerada apenas uma das visitas realizadas nas unidades de saúde daqueles municípios.

Em relação à avaliação de processo, é importante considerar que, nas unidades de saúde de dois municípios, Agrestina e Passira, houve apenas uma visita de seguimento. Nesses, os principais fatores que contribuíram para a classificação no nível insatisfatório foram os escores atingidos referentes à alimentação e à necessidade de retorno dos pacientes, à análise da compreensão por parte das mães ou acompanhantes das crianças e, ainda, ao baixo índi- ce de seguimento da sistematização do atendimento previsto.

No Município de Saloá, foram realizadas duas visitas. Na primeira estabeleceu-se apenas uma discussão sobre os problemas encontrados, uma vez que os profissionais não estavam seguindo a normatização da estratégia, embora na unidade de saúde houvesse disponibilidade dos insumos, equipamentos e medicamentos previstos para a implantação da mesma. Na segunda visita, os profissionais já seguiam a normatização e, mesmo tendo apresentado alguma dificuldade em seguir a sistematização do atendimento, contribuíram de forma importante para que o município atingisse um nível aceitável na determinação do grau de implantação. Posteriormente, porém, houve interrupção no desenvolvimento da estratégia, tendo em vista a mudança de profissionais da equipe de saúde.

Também nos municípios de Água Preta, Bezerros e Garanhuns foram realizadas apenas duas visitas de seguimento. Entre esses, apenas Bezerros apresentou grau de implantação insatisfatório em uma das visitas de seguimento. A classificação obtida deve-se principalmente aos baixos escores individuais, obtidos nas variáveis que representam a verificação da compreensão das orientações fornecidas e a não disponibilidade, em uma das unidades, de vários medicamentos padronizados pela estratégia.

Os municípios de Brejão, Cabo de Santo Agostinho, Caetés e Caruaru, receberam três visitas. Entre esses, apenas o segundo apresentou classificação no nível insatisfatório e dessa feita, em duas oportunidades, sendo que naquela em que obteve classificação no nível aceitável, esta se deu mediante escore bem próximo do nível imediatamente inferior. Os principais fatores observados para essa situação são aqueles concernentes às recomendações sobre imunização, alimentação, retorno à unidade de saúde e à compreensão sobre estas recomendações e sobre os problemas e os tratamentos observados. Além destes, a baixa adesão dos profissionais em seguir a sistematização proposta pela estratégia, a constante ausência de medicamentos padronizados e a falta de insumos nas unidades como os necessários para a terapia de reidratação oral (TRO), fichas de atendimento, além de termômetro e cartão da criança, foram determinantes para a classificação alcançada.

Os indicadores de processo normatizados pelo MS e integrantes do Manual de Acompanhamento e Avaliação da AIDPI, permitem classificar os municípios por intermédio da média aritmética dos percentuais obtidos para cada indicador (MS, 2000). Foi importante utilizar 
esse instrumento, principalmente como forma de validar o sistema de escores construído. Os indicadores foram aplicados para cada visita de seguimento nos dez municípios estudados, obtendo-se assim classificações específicas que puderam ser comparadas com as anteriormente descritas, como observa-se na Tabela 2.

$\mathrm{Na}$ análise do contexto ao nível local, aferese algumas características comuns ao conjunto dos municípios que vêm se somar à situação de habilitação de acordo com a Norma Operacional Básica 1996 (NOB-96) (MS, 1996). Destacase a questão dos recursos financeiros empregados para o desenvolvimento da estratégia em que, na totalidade dos casos, os municípios tinham promovido o aporte apenas de insumos, incluindo-se aí medicamentos e impressos, no período do estudo. Após esse período, entretanto, o Município de Caruaru veio a financiar parte da capacitação de seus profissionais, promovendo assim um avanço significativo na implementação da estratégia que, até setembro de 2000 , fazia parte da rotina de 25 das 26 equipes do PSF em funcionamento no município. $\mathrm{O}$ restante deles dependia de recursos estaduais e federais para a capacitação dos profissionais. Outro dado comum é que todos dispunham de apenas um relatório de supervisão, este referente à primeira visita de seguimento realizada em meados de 1998.

Outra característica importante é a formação dos gestores mais diretamente envolvidos, uma vez que todos tinham formação superior, sendo que entre os secretários municipais de saúde, apenas os de Agrestina e Passira não eram profissionais da área de saúde, e os dos municípios de Brejão, Cabo de Santo Agostinho e Caetés apresentavam formação específica na área de saúde pública. Os coordenadores do PSF de todos os municípios tinham nível superior e todos na área de saúde, sendo que os de Bezerros, Caetés, Caruaru e Saloá tinham formação em saúde pública. Apenas em dois municípios, Garanhuns e Saloá, os coordenadores do PSF não coordenavam a estratégia AIDPI no nível local.

Ainda em relação aos gestores, o Município do Cabo de Santo Agostinho foi o que mais apresentou mudanças, tendo sido substituídos os três principais atores em relação à estratégia: o secretário de saúde, o coordenador do PSF e o coordenador da AIDPI. Outros dois municípios apresentaram mudanças: Garanhuns que trocou o secretário de saúde e o coordenador do PSF e Saloá que mudou o coordenador do PSF e o da AIDPI.

Os municípios de Bezerros, Brejão e Garanhuns promoveram alguma discussão no Conselho Municipal de Saúde, pois foram encon-
Tabela 2

Comparação da classificação dos municípios segundo o grau de implantação, quando aplicados o sistema de escores e os indicadores de processo, 1998-1999.

\begin{tabular}{|c|c|c|}
\hline \multirow[t]{2}{*}{ Município } & \multicolumn{2}{|c|}{ 1으 Seguimento } \\
\hline & Sistema de escores & Indicadores de processo \\
\hline Agrestina & 7,2 & 7,8 \\
\hline Água Preta & 8,4 & 8,4 \\
\hline Bezerros & 8,7 & 8,1 \\
\hline Brejão & 9,5 & 9,2 \\
\hline Cabo & 8,1 & 7,7 \\
\hline Caetés & 9,7 & 8,9 \\
\hline Caruaru & 8,9 & 9,0 \\
\hline Garanhuns & 9,0 & 8,2 \\
\hline Passira & 6,8 & 7,3 \\
\hline Saloá & 3,3 & 4,3 \\
\hline \multirow[t]{2}{*}{ Município } & \multicolumn{2}{|c|}{ 2o Seguimento } \\
\hline & Sistema de escores & Indicadores de processo \\
\hline Agrestina & - & - \\
\hline Água Preta & 9,3 & 10,0 \\
\hline Bezerros & 7,9 & 7,9 \\
\hline Brejão & 9,0 & 10,0 \\
\hline Cabo & 7,7 & 7,9 \\
\hline Caetés & 8,9 & 9,3 \\
\hline Caruaru & 9,4 & 9,2 \\
\hline Garanhuns & 9,3 & 8,5 \\
\hline Passira & - & - \\
\hline Saloá & 8,2 & 8,2 \\
\hline
\end{tabular}

\begin{tabular}{lcc} 
Município & \multicolumn{2}{c}{ 3o Seguimento } \\
& Sistema de escores & Indicadores de processo \\
\hline Agrestina & - & - \\
Água Preta & - & - \\
Bezerros & - & - \\
Brejão & 9,8 & 10,0 \\
Cabo & 7,1 & 8,2 \\
Caetés & 9,3 & 8,6 \\
Caruaru & 9,6 & 9,9 \\
Garanhuns & - & - \\
Passira & - & - \\
Saloá & - & -
\end{tabular}

\begin{tabular}{lcc}
\hline Município & \multicolumn{2}{c}{ Média dos três seguimentos } \\
Indicadores de processo \\
\hline Agrestina & Sistema de escores & 7,8 \\
Água Preta & 7,2 & 9,1 \\
Bezerros & 8,8 & 8,0 \\
Brejão & 8,3 & 9,7 \\
Cabo & 9,4 & 7,9 \\
Caetés & 7,6 & 8,9 \\
Caruaru & 9,3 & 9,4 \\
Garanhuns & 9,3 & 8,3 \\
Passira & 9,1 & 7,3 \\
Saloá & 6,8 & 6,2 \\
\hline
\end{tabular}


trados registros em atas dos Conselhos sobre a AIDPI, e apenas Garanhuns e Caetés formularam planos de trabalho que contemplavam a previsão de implantação da estratégia em novas unidades.

Quando se observa a influência dos profissionais médicos e enfermeiros integrantes das equipes de saúde e foco determinante do processo avaliativo, deve-se começar por assinalar o percentual de mudança dos mesmos nos diversos municípios: $50 \%$ de substituição em Bezerros e Saloá, 25\% em Brejão e 12,5\% em Água Preta. Em Agrestina e Passira, como já assinalado, as equipes do PSF foram desfeitas antes da segunda visita de seguimento e nos demais municípios não foram relatadas mudanças em relação a esses profissionais.

Na Tabela, 3 apresentam-se as considerações mais freqüentes referidas por esses profissionais nas entrevistas realizadas durante as visitas de seguimento.

Na Tabela 4, têm-se um demonstrativo dos principais resultados das entrevistas com os coordenadores locais do PSF, associados ao grau de implantação observado nos municípios. Promove-se, ainda, a desagregação da nota final do caso e dos insumos, buscando tornar mais evidentes as associações procuradas.

Essa desagregação permite destacar que, das cinco situações onde os municípios apresentam grau de implantação no nível insatisfatório, em três delas (Agrestina - 1o seguimento e Cabo de Santo Agostinho - 2o e 3o seguimentos) isso se dá decorrente, principalmente, da nota final do caso $(6,8 ; 7,2$ e 6,8). Nas outras duas (Bezerros 2o seguimento e Passira - 1o), a influência da nota final do caso $(8,0$ e 6,9) e da nota final dos insumos $(7,9$ e 6,5$)$ ocorre de forma semelhante.

\section{Discussão e conclusão}

A análise do grau de implantação da estratégia nos municípios estudados, permite observar o alto grau de coerência entre os dois métodos utilizados, o sistema de escores e os indicadores de processo, tanto nas comparações específicas por visita de seguimento, como quando são comparados os escores médios dos três momentos do estudo.

No Estado de Pernambuco, existe um contexto favorável à implantação da estratégia, considerando desde a observação de como se iniciou o processo, ao se ter escolhido as unidades do PSF, já detentoras de uma normatização própria para condições estruturais, de condutas e método de trabalho em equipe multidisciplinar e com integração à comunidade, até o esforço desenvolvido pela SES-PE estimulando a habilitação de $100 \%$ dos municípios na NOB-96, e possibilitando maior autonomia na gestão dos recursos e no planejamento e execução das políticas setoriais locais (MS, 1996; SES-PE, 1998a). Dos dez municípios estudados, três encontramse no nível de gestão plena do sistema municipal, e os outros sete na gestão plena da atenção básica.

O incremento do PSF no estado e o financiamento garantido por parte dos níveis estadual e federal para os cursos de capacitação da estratégia, contribuíram de forma importante para isso. Basta verificar que nos 10 municípios selecionados havia, até setembro de 2000, 108 equipes do PSF e, dessas, 64 encontravam-se capacitadas em AIDPI, quando esse número era de 33 e todas treinadas, em 1998, período a partir do qual se iniciou este estudo (Felisberto, 2000; SES-PE, 1998b).

Aspecto de fundamental importância a ser discutido, são as referências dos profissionais avaliados em relação ao que consideram pontos positivos e negativos da estratégia AIDPI. Denis \& Champagne (1997:78), quando discorrem sobre a influência dos médicos em suas considerações metodológicas para a análise de implantação, afirmam: "o desenvolvimento das práticas multidisciplinares é fortemente ligado à propensão do médico a envolver-se em tais atividades, o que por sua vez depende do modelo de intervenção defendido por este ator. De fato, os médicos têm opiniões diversas sobre a utilidade dos outros atores no processo de execução de cuidados..."

As práticas profissionais das equipes do PSF já baseadas no trabalho do médico e do enfermeiro, e o método de operacionalização da AIDPI, contribuem para explicar que em $100 \%$ dos municípios estudados os profissionais referiram entre os aspectos positivos a adesão da equipe. Além disso, o aumento da capacidade resolutiva da mesma, foi citado em oito dos dez municípios. Esses resultados atendem ao modelo político e contingente defendido pelos autores acima citados, e adotados neste estudo como referencial para análise de implantação, assim como constituem-se em características contextuais que podem influenciar o grau de implantação segundo o modelo político em que aquele se baseia, sugerido por vários autores e sistematizado por Denis \& Champagne (1997).

Ainda, segundo Denis \& Champagne (1997), há um outro modelo em que se baseia o modelo político e contingente, é o modelo estrutural, segundo o qual uma série de características referentes aos atributos organizacionais e aos dos gestores podem ter influência na determinação 
Tabela 3

Demonstrativo dos resultados das entrevistas com os profissionais de saúde das unidades avaliadas, 1998-1999.

\begin{tabular}{|c|c|c|}
\hline Município & Aspectos positivos & Aspectos negativos \\
\hline Agrestina & Adesão da equipe & $\begin{array}{l}\text { Falta de medicamentos padronizados } \\
\text { Pouca sensibilidade dos gestores }\end{array}$ \\
\hline Água Preta & $\begin{array}{l}\text { Adesão da equipe } \\
\text { Diminuição dos encaminhamentos } \\
\text { para internação }\end{array}$ & $\begin{array}{l}\text { Falta de medicamentos padronizados } \\
\text { Dificuldade para referência de pacientes } \\
\text { Demanda excessiva }\end{array}$ \\
\hline Bezerros & $\begin{array}{l}\text { Adesão da equipe } \\
\text { Aumento da capacidade resolutiva } \\
\text { da unidade }\end{array}$ & $\begin{array}{l}\text { Falta de medicamentos padronizados } \\
\text { Dificuldade para referência de pacientes } \\
\text { Dificuldade para viabilizar o retorno dos pacientes }\end{array}$ \\
\hline Brejão & $\begin{array}{l}\text { Adesão da equipe } \\
\text { Apoio do Secretário de Saúde } \\
\text { Aumento da capacidade resolutiva } \\
\text { da unidade } \\
\text { Instalações, equipamentos e insumos } \\
\text { adequados }\end{array}$ & Dificuldade para referência de pacientes \\
\hline $\begin{array}{l}\text { Cabo de } \\
\text { Santo Agostinho }\end{array}$ & $\begin{array}{l}\text { Adesão da equipe } \\
\text { Aumento da capacidade resolutiva } \\
\text { da unidade }\end{array}$ & $\begin{array}{l}\text { Falta de material } \\
\text { Falta de medicamentos } \\
\text { padronizados } \\
\text { Dificuldade para referência de pacientes } \\
\text { Demanda excessiva } \\
\text { Não valorização da estratégia } \\
\text { pelo nível central }\end{array}$ \\
\hline Caetés & $\begin{array}{l}\text { Adesão da equipe } \\
\text { Integração da equipe com } \\
\text { a comunidade } \\
\text { Unidades com instalações adequadas } \\
\text { Aumento da capacidade resolutiva } \\
\text { da unidade } \\
\text { Intercâmbio de experiências entre } \\
\text { as equipes do município }\end{array}$ & $\begin{array}{l}\text { Falta de material } \\
\text { Dificuldade para referência de pacientes }\end{array}$ \\
\hline Caruaru & $\begin{array}{l}\text { Adesão da equipe } \\
\text { Aumento da capacidade resolutiva } \\
\text { da unidade } \\
\text { Apoio dos gestores }\end{array}$ & $\begin{array}{l}\text { Falta de medicamentos padronizados } \\
\text { Dificuldade para referência de pacientes } \\
\text { Dificuldades na orientação alimentar } \\
\text { Dificuldade para viabilizar o retorno dos pacientes }\end{array}$ \\
\hline Garanhuns & $\begin{array}{l}\text { Adesão da equipe } \\
\text { Integração da equipe } \\
\text { Aumento da capacidade resolutiva } \\
\text { da unidade }\end{array}$ & $\begin{array}{l}\text { Falta de medicamentos padronizados } \\
\text { Dificuldade para referência de pacientes }\end{array}$ \\
\hline Passira & $\begin{array}{l}\text { Adesão da equipe } \\
\text { Integração da equipe com } \\
\text { a comunidade } \\
\text { Satisfação do usuário } \\
\text { Aumento da capacidade resolutiva } \\
\text { da unidade }\end{array}$ & $\begin{array}{l}\text { Falta de material } \\
\text { Falta de medicamentos padronizados } \\
\text { Dificuldade para referência de pacientes } \\
\text { Instalações inadequadas }\end{array}$ \\
\hline Saloá & $\begin{array}{l}\text { Adesão da equipe } \\
\text { Integração da equipe }\end{array}$ & $\begin{array}{l}\text { Falta de material } \\
\text { Dificuldade para referência de pacientes } \\
\text { Dificuldade na organização da unidade } \\
\text { Dificuldade para viabilizar o retorno dos pacientes } \\
\text { Falta de apoio e sensibilização dos gestores }\end{array}$ \\
\hline
\end{tabular}


Demonstrativo dos principais resultados das entrevistas com os coordenadores municipais do Programa Saúde da Família (PSF), associados ao grau de implantação observado nos municípios.

\begin{tabular}{|c|c|c|c|c|c|c|c|c|c|}
\hline \multirow[t]{2}{*}{ Municípios } & \multicolumn{9}{|c|}{ Variáveis } \\
\hline & $\begin{array}{l}\text { Gestão } \\
\text { Municipal } \\
\text { NOB-96 }\end{array}$ & $\begin{array}{l}\text { Formação } \\
\text { SMS }\end{array}$ & $\begin{array}{l}\text { Saúde } \\
\text { pública }\end{array}$ & $\begin{array}{l}\text { Formação } \\
\text { Coordenador(a) } \\
\text { PSF }\end{array}$ & $\begin{array}{l}\text { Saúde } \\
\text { Pública }\end{array}$ & $\begin{array}{l}\text { Registro em } \\
\text { atas do CMS }\end{array}$ & $\begin{array}{l}\text { Plano de } \\
\text { trabalho }\end{array}$ & $\begin{array}{l}\text { Mudança } \\
\text { de SMS }\end{array}$ & $\begin{array}{l}\text { Mudança } \\
\text { Coordenador(a) } \\
\text { AIDPI }\end{array}$ \\
\hline Agrestina & PAB & NS(Hist) & $\mathrm{N}$ & NS(Enf) & $\mathrm{N}$ & $\mathrm{N}$ & $\mathrm{N}$ & $\mathrm{N}$ & $\mathrm{N}$ \\
\hline Água Preta & PAB & NS(Med) & $\mathrm{N}$ & NS(Med) & $\mathrm{N}$ & NSI & NSI & $\mathrm{N}$ & $N$ \\
\hline Bezerros & PSM & NS(Med) & $\mathrm{N}$ & NS(Med) & $\mathrm{S}$ & $\mathrm{S}$ & $\mathrm{N}$ & $\mathrm{N}$ & $N$ \\
\hline Brejão & PAB & NS(Med) & $\mathrm{S}$ & NS(Enf) & $\mathrm{N}$ & $\mathrm{S}$ & $\mathrm{N}$ & $\mathrm{N}$ & $\mathrm{N}$ \\
\hline Cabo & PSM & NS(Med) & $\mathrm{S}$ & NS(Méd) & $\mathrm{N}$ & $\mathrm{N}$ & $\mathrm{N}$ & $\mathrm{S}$ & $\mathrm{S}$ \\
\hline Caetés & PAB & NS(Psic) & $\mathrm{S}$ & NS(Psic) & $\mathrm{S}$ & $N$ & $\mathrm{~S}$ & $\mathrm{~N}$ & $\mathrm{~N}$ \\
\hline Caruaru & PSM & NS(Med) & $\mathrm{N}$ & NS(Enf) & $\mathrm{S}$ & NSI & $\mathrm{N}$ & $\mathrm{N}$ & $N$ \\
\hline Garanhuns & PAB & NS(Biom) & $\mathrm{N}$ & NS(Odon) & $\mathrm{N}$ & $\mathrm{S}$ & $\mathrm{S}$ & $\mathrm{S}$ & $\mathrm{N}$ \\
\hline Passira & PAB & $\mathrm{NS}(\mathrm{Edu})$ & $\mathrm{N}$ & NS(Enf) & $\mathrm{N}$ & $\mathrm{N}$ & $\mathrm{N}$ & $\mathrm{N}$ & $\mathrm{N}$ \\
\hline Saloá & PAB & NS(Med) & $\mathrm{N}$ & NS(Psic) & $\mathrm{S}$ & $\mathrm{N}$ & $\mathrm{N}$ & $\mathrm{N}$ & $\mathrm{S}$ \\
\hline \multirow[t]{3}{*}{ Municípios } & \multicolumn{9}{|c|}{ Grau de implantação } \\
\hline & \multicolumn{3}{|c|}{ 1ㅇ seguimento } & \multicolumn{3}{|c|}{ 2o seguimento } & \multicolumn{3}{|c|}{ 3o seguimento } \\
\hline & NFC & Gl & $\mathrm{NFI}$ & NFC & GI & $\mathrm{NFI}$ & NFC & GI & $\mathrm{NFI}$ \\
\hline Agrestina & 6,8 & 1 & 8,2 & & - & & & - & \\
\hline Água Preta & 8,9 & A & 7,5 & 9,0 & A & 9,8 & & - & \\
\hline Bezerros & 8,4 & A & 9,2 & 8,0 & 1 & 7,9 & & - & \\
\hline Brejão & 9,3 & A & 10,0 & 8,6 & A & 10,0 & 9,8 & A & 10,0 \\
\hline Cabo & 7,9 & A & 8,5 & 7,2 & 1 & 8,7 & 6,8 & I & 7,8 \\
\hline Caetés & 9,6 & A & 9,8 & 9,0 & A & 8,8 & 9,3 & A & 9,3 \\
\hline Caruaru & 8,9 & A & 9,0 & 9,2 & A & 9,9 & 9,4 & $A$ & 9,8 \\
\hline Garanhuns & 9 & A & 8,9 & 9,6 & A & 8,6 & & - & \\
\hline Passira & 6,9 & 1 & 6,5 & & - & & & - & \\
\hline Saloá & 0,0 & C & 10,0 & 7,4 & A & 7,4 & & - & \\
\hline
\end{tabular}

SMS = Secretário Municipal de Saúde; CMS = Conselho Municipal de Saúde; AIDPI = Atenção integrada às doenças prevalentes da infância; $\mathrm{PAB}=$ Plena da Atenção Básica; PSM = Plena do Sistema Municipal; NS = Nível Superior; $\mathrm{S}=\mathrm{Sim} ; \mathrm{N}=\mathrm{Não}$; NSI = Não sabe informar;

$\mathrm{NFI}=$ Nota final do insumo; NFC = Nota final do caso; GI = Grau de implantação; $\mathrm{A}=$ Aceitável; I = Insatisfatório; $\mathrm{C}=$ Critíco; Hist = Licenciatura em História; Med = Medicina; Psic = Psicologia; Biom = Ciências Biomédicas; Edu = Pedagogia; Odon = Odontologia; Enf = Enfermagem.

do grau de implantação de uma intervenção. Eles sistematizam uma indagação atendida favoravelmente pelo processo de implantação da AIDPI no estado de Pernambuco: "As características dos gestores, do ambiente e da organização favorecem a implantação da intervenção?" (Denis \& Champagne, 1997:69).

Entretanto, este estudo revela não haver associação direta entre as variáveis escolhidas para avaliação do contexto organizacional no nível municipal e os diversos graus de implantação, quando se procura estabelecer uma associação entre estes de forma desagregada, verificando a nota final do caso e a nota final dos insumos por visita de seguimento e por município. Isso pode ser explicado por uma possível insuficiên- cia de sensibilidade dessas variáveis em expressar as diferentes dimensões do grau de implantação, associado ao fato de que o conjunto dos municípios avaliados sofreram influência semelhante dos fatores contextuais discutidos anteriormente.

Conclui-se, também, que o grau de implantação encontrado apresentava-se no nível aceitável na maioria dos municípios e, naqueles onde se identificou o nível insatisfatório, isso se deu em decorrência, principalmente, de fatores relacionados ao manejo de casos e, mais freqüentemente, ao componente que reflete a interação entre a prática profissional e o entendimento dessa prática pelo usuário. 


\section{Referências}

BENGUIGUI, Y.; LAND, S.; PAGANINI, J. M. \& YUNES, J., (org.), 1997. Ações de Saúde Materno-Infantil a Nível Local: Segundo as Metas da Cúpula Mundial em Favor da Infância. Washington, DC: Organização Pan-Americana da Saúde.

COSENDEY, M. A. E., 2000. Análise de Implantação do Programa de Farmácia Básica: Um Estudo Multicêntrico em Cinco Estados do Brasil. Tese de Doutorado, Rio de Janeiro: Escola Nacional de Saúde Pública, Fundação Oswaldo Cruz.

CONTANDRIOPOULOS, A.-P.; CHAMPAGNE, F.; POTVIN, L.; DENIS, J.-L. \& BOYLE, P., 1994. Saber Preparar uma Pesquisa. Rio de Janeiro: ABRASCO/ São Paulo: Editora Hucitec.

DEAN, A. G.; DEAN, J. A.; COULOMBIER, D.; BRENDEL, K. A.; SMITH, D. C.; BURTON, A. H.; DICKER, R. C.; SULLIVAN, K.; FAGAN, R. F. \& ARNER, T. G., 1994. Epi Info, Version 6: A Word Processing, Database and Statistics Program for Epidemiology on Microcomputers. Atlanta: Centers for Disease Control and Prevention.

DENIS, J. L. \& CHAMPAGNE, F., 1997. Análise de implantação. In: Avaliação em Saúde: Dos Modelos Conceituais à Prática na Análise da Implantação de Programas (Z. M. A. Hartz, org.), pp. 49-88, Rio de Janeiro: Editora Fiocruz/ABRASCO.

DONABEDIAN, A., 1990. The seven pillars of quality. Archives of Pathology and Laboratory Medicine, 114:1115-1118.

FELISBERTO, E.; CARVALHO, E. F. \& SAMICO, I., 2000. Estratégia da Atenção Integrada às Doenças Prevalentes da Infância - Considerações sobre o processo de implantação. Revista do IMIP, 14:24-31.

HARTZ, Z. M. A. (org.), 1997. Avaliação em Saúde: Dos Modelos Conceituais à Prática na Análise da Implantação de Programas. Rio de Janeiro: Editora Fiocruz/ABRASCO.

MS (Ministério da Saúde), 1996. Norma Operacional Básica. Brasília: MS.
MS (Ministério da Saúde), 2000. Manual de Acompanhamento e Avaliação da AIDPI: Módulo 9. Brasília: MS.

OJEDA, N. S., 1992. Evaluation of maternal and child health services in Latin America. World Health Forum, 13:139-142.

OPAS (Organização Pan-Americana da Saúde)/OMS (Organização Mundial da Saúde), 1987. Condições de Eficiência dos Serviços de Atenção Materno-Infantil. Brasília: OPAS.

OPS (Organización Panamericana de la Salud), 1998. La atención integrada a las enfermedades prevalentes de la infancia (AIEPI) en países de América Latina. Informe de la Reunión de evaluacíon y planificacíon del proyecto USAID/LAC-OPS-BASICS. Quito: OPS.

SAS (Secretaria de Assistência à Saúde), 1994. Roteiro de Avaliação dos Programas PAISM/PAISC PROSAD. Brasília: Ministério da Saúde. (mimeo.)

SES-PE (Secretaria Estadual de Saúde de Pernambuco), 1996. Avaliação das Ações de Atenção Integral à Saúde da Criança: Situação Atual - Grau de Implantação e de Gestão Municipal. Cadernos de Avaliação, Série Projeto Salva Vidas 4. Recife: SESPE.

SES-PE (Secretaria Estadual de Saúde de Pernambuco), 1998a. Processo de Implantação da Estratégia AIDPI em Pernambuco: Uma Experiência de Parceria com o Instituto Materno Infantil de Pernambuco. Cadernos de Avaliação, Série Projeto Salva Vidas 14. Recife: SES-PE.

SES-PE (Secretaria Estadual de Saúde de Pernambuco), 1998b. Relatório de Gestão. Recife: SES-PE.

VUORI, H., 1988. A Qualidade da Saúde. Rio de Janeiro: Escola Nacional de Saúde Pública, Fundação Oswaldo Cruz. (mimeo.)

WHO (World Health Organization), 1997. Integrated management of childhood illness: Global status of implementation. News on Health Care in Developing Countries, 11:4-9.

Recebido em 19 de setembro de 2001

Versão final reapresentada em 8 de abril de 2002

Aprovado em 18 de junho de 2002 